\title{
Coherent quantum phenomena in a normal cylindrical conductor with a superconducting coating
}

\author{
G. A. Gogadze \\ Department of Applied Physics, Chalmers University of Technology and Göteborg University, \\ SE-41296 Göteborg, Sweden and \\ B. I. Verkin Institute for Low Temperature Physics and Engineering of the National Academy of Science of Ukraine, \\ 47 Lenin Ave., 61103 Kharkov, Ukraine \\ E-mail: gogadze@ilt.kharkov.ua \\ R. I. Shekhter and M. Jonson \\ Department of Applied Physics, Chalmers University of the Technology and Göteborg University \\ SE-41296 Göteborg, Sweden
}

Received April 28, 2001

\begin{abstract}
The thermodynamic properties of a mesoscopic-size simply connected cylindrical normal metal in good metallic contact with superconducting banks are studied theoretically. It is commonly accepted that if the superconductor thickness is quite small (of the order of the coherence length), as is assumed to be the case here, a vector potential field, whose value can be varied, exists inside the normal layer. It is further assumed that the quasiparticles with energy $E<\Delta$ ( $2 \Delta$ is the superconducting gap) move ballistically through the normal metal and undergo Andreev scattering caused by the off-diagonal potential of the superconductor. An equation is obtained within the multidimensional quasiclassical method which permits us to determine the spectrum of the Andreev levels and to calculate the density of states of the system in question. It is shown that the Andreev levels shift as the trapped flux $\Phi$ changes inside the normal conductor. At a certain flux value they coincide with the Fermi level. A resonance spike in the density of states $v(E)$ appears in this case, since near $E=0$ there is strong degeneracy of the quasiparticle states in respect to the quantum number $q$ characterizing their motion along the cylinder axis. As a result, a macroscopic number of $q$ states contribute to the amplitude of the effect. As the flux is increased, the density of states $v(E)$ behaves as a stepwise function of $\Phi$. The distance between the steps is equal to the superconducting flux quantum $h c / 2 e$.
\end{abstract}

PACS: 72.10.-d

\section{Introduction}

Quantum interference phenomena in condensed matter were first observed in superconductors. Soon after the microscopic Bardeen-Cooper-Schrieffer theory of superconductivity [1] appeared, the effect of flux quantization was detected experimentally in a superconducting ring [2,3]. This effect had been predicted previously by F. London [4] who showed that in a doubly connected superconductor a certain quantity (fluxoid) could take only quantized values. The fluxoid is determined by the sum of magnetic fluxes through the superconductor cross section plus an integral term dependent on the superfluid current. In a bulk superconductor with an orifice, flux quantization exists in its direct meaning since the magnetic field does not penetrate inside the superconductor. The integration path used to calculate the integral fluxoid term can be chosen to lie rather deep inside the metal, where the superfluid current is zero.

For a thin-wall cylinder the flux can take any value, and flux quantization shows up as oscillations of the current circulating over the cylinder surface. The oscillation period of the magnetic field flux is equal to one superconducting flux quantum $\Phi_{0}=h c / 2 e$.

It was thought for a long time that flux quantization could exist only in superconductors and was associated with (i) the sensitivity of quantum electron states to the vector potential field (the Aharonov-Bohm effect [5]), and (ii) the presence of an 
off-diagonal long range order in superconductors [6]. In 1970 Kulik published an important theoretical paper [7] describing the behavior of a thin-wall cylindrical normal conductor in the vector potential field excited by an inserted solenoid. That was the first evidence suggesting that coherent quantum properties are possible in normal (nonsuperconducting) systems of quite small (though «macroscopic» in atomic scale) dimensions at low temperatures. It turns out that the magnetic moment of such a system is an oscillatory function of the magnetic flux through the cylinder orifice, and the oscillation period is equal to one flux quantum of the normal metal $h c / e$. The quantization effect takes place in the absence of off-diagonal longrange order.

Reference [7] has stimulated much interest in quantum interference effects in normal condensed media. It has become clear that the AharonovBohm effect is possible in solids because the electron retains its «phase memory» over a finite length $\xi_{N}=\hbar v_{F} / \pi T$, which has the meaning of a coherence length in a system with a disturbed longrange order $\left(v_{F}\right.$ is the Fermi velocity, $T$ is the temperature). With an infinitely long electron mean free path and at temperatures $T \sim 1 \mathrm{~K}, \xi_{N}$ is about $10^{-3} \mathrm{~cm}$, and coherent quantum phenomena become possible in normal conductors whose transverse dimensions are of the order of $\xi_{N}$. However, because a doubly connected conductor should have a very small cross section, the idea of an experiment with an inserted solenoid seemed problematic. The need arose to study coherent quantum effects in simply connected cylindrical conductors in a magnetic field and to analyze the influence of surface and bulk collisions on the oscillation amplitudes. It was shown for the first time in 1972 [8] that flux quantization is possible in pure cylindrical solid conductors placed in a weak longitudinal magnetic field (such that the cyclotron radius much exceeds that of the cylinder). The oscillations of thermodynamic quantities in simply connected normal conductors present a more complicated case, which involves two oscillatory components in their magnetic-field dependence. One of them is intricately dependent on the number of the harmonics and the magnetic field and is correlated with the electrons of the central Fermi surface section. The other is characterized by a universal (independent of the dispersion relations for the carriers) period of the magnetic field flux $h c / e$. The latter oscillations are caused by the skipping-orbit electrons localized in a thin layer (with a thickness of about the de Broglie electron wavelength) near the cylinder surface. The effect is due to size quantization and to the sensitivity of the phase of the electron wave function to the vector potential field.

The spectrum of magnetic surface levels responsible for the coherent phenomena in a normal cylindrical conductor in a weak magnetic field was obtained in Ref. 8. The nature of these levels is different from what was described by Nee and Prange [9]. The magnetic surface levels at a flat boundary are genetically related to the magnetic field and vanish when the field is zero. In a cylinder the magnetic surface levels are formed by the sample boundary, but the magnetic flux is included directly in the expression for the electronic energy spectrum. This expression has been used to calculate a number of specific effects $[8,10-14]$. In particular, the effect of doubling the oscillation period of the critical temperature has been calculated for a superconducting hollow cylinder with allowance for the quantization of single-particle excitations [15]. When the electron spectrum is smeared, a characteristic period of the Little-Parks effect [16-18] equal to the superconducting flux quantum $h c / 2 e$ appears. We should also mention the AharonovBohm effect for electrons localized above liquid helium covering the surface of a dielectric cylinder with the magnetic field applied along its axis [19]. In such a system the electrons which obey Boltzmann statistics move along quasiclassical trajectories and acquire equal increments of wave function phase. As a result the system experiences magnetic-moment oscillations with a universal period $h c / e$ of magnetic flux.

The effect of flux quantization in a normal, simply connected cylindrical conductor was experimentally detected by Brandt, Gitsu, Nikolaeva and Ponomarev [20] (see also Refs. 21 and 22) while investigating the longitudinal magnetoresistance of pure Bi single crystals. This was the first observation of an interference effect related to flux quantization in nonsuperconducting condensed media.

An important distinctive feature of flux quantization in normal metals is the presence of the factor $\cos \left(2 \pi k_{F} R+\alpha\right)$ in the oscillation amplitude ( $\hbar k_{F}$ is the Fermi momentum, $\alpha$ is a auxiliary phase). The implication is that in normal metals a variation of $R$ (fluctuation) by a value of the order of the atomic spacing causes a $100 \%$ change in the effect [23]. In this case the flux quantization effect should vanish after oscillation amplitude averaging along the cylinder axis. For this reason the effect was observed experimentally in semimetals, where fluctuations on the scale of the atomic radius are unimportant for 
the amplitude of flux quantization because of the smallness of the effective mass of the carriers. In currently accepted terms, this feature of flux quantization amplitudes in normal metals reflects the mesoscopic character of the effects [24].

The concepts of interference phenomena in condensed media have been further extended to dirty systems. Now we understand that because of its mesoscopic character, the flux quantization effect with period $h c / e$ is only possible in small metallic rings where no averaging over different impurity configurations is required $[25,26]$. In this case we are dealing with interference effects in the conductivity of low-dimensional conductors, since with increasing impurity concentration the oscillation amplitude of the thermodynamic (and kinetic) magnitudes decreases exponentially. In rather long dirty cylindrical conductors (or a large number of rings) the $h c / e$ quantization disappears.

Along with mesoscopic flux quantization in normal metals, there is another important class of interference phenomena induced by weak localization. These phenomena were predicted theoretically by Altshuler, Aronov, and Spivak [27] in 1981 when they were calculating the quantum correction to the conductivity of a metallic thin-wall cylinder in a longitudinal magnetic field. The quantum correction evolves from interference of the wave functions of the particles moving along different trajectories. For arbitrary trajectories the difference between the wave function phases is a random value, and the wave interference (after trajectory averaging) makes zero contribution to the conductivity. The sole exceptions are self-crossing trajectories, when each trajectory can be related to its time-reversed counterpart. The electron on the reversed trajectory experiences scatterings identical to those on the initial trajectory but in a reversed sequence.

When the flux is trapped in the cylinder hole, the phase difference of the interfering waves can be varied, which makes the magnetoresistance oscillate with the magnetic flux period $h c / 2 e$ [27].

To observe this effect, the cylinder radius should not exceed the distance along which the electron retains its «phase memory». This scale is $L_{\Phi}=$ $=\left(D \tau_{\Phi}\right)^{1 / 2}$, where $D$ is the diffusion coefficient, and $\tau_{\Phi}$ is the phase break time, which is dependent on temperature. Magnetoresistance oscillations in dirty metallic cylinders with flux period $h c / 2 e$ were first observed experimentally by Yu. Sharvin and D. Sharvin $[28,29]$. These experiments prove that elastic scattering does not disturb the phase coherence of the electrons. Only inelastic processes (changing the energy condition of the electron) can influence the «phase memory» by smearing the interference situation. The effect does not disappear on averaging over the impurity distributions. In contrast to the mesoscopic effects, the $h c / 2 e$ oscillations vanish in rather weak magnetic fields.

The experimental detection of the AharonovBohm effect in condensed media has become possible due to the recent advance in the technology for producing metallic, semimetallic and semiconducting cylinders and rings. In his experiments Brandt et al. [20-22] used casting technology to produce Bi single crystals from the liquid phase. Cylindrical samples of high purity were obtained under a glass coating which was not removed during the measurement. The coating reinforced the samples mechanically and protected their surfaces from environmental influences. Experimental measurements on such samples ensured reproducible results even several years later (private communication by Ya. G. Ponomarev).

Observation of interference effects in disordered media has become possible due to miniaturization of structures and to the progress in microelectronic lithography. The experiments performed by Yu. Sharvin and D. Sharvin $[28,29]$, Webb et al. [25], and Chandrasekhar et al. [26] have clarified the possibility of making quite small instruments taking advantage of new mesoscopic features of the physics of condensed matter.

Quantum interference phenomena have been discussed in detail in a number of surveys [ 30-33, 24] and articles [34-36].

This study is concentrated on coherent quantum phenomena in a mesoscopic-size cylindrical normal conductor contiguous with a superconductor.

In recent years there has been extensive work in the field of mesoscopic systems, including superconducting structures in proximity with normal metals. The experimental work [37] discussed the magnetic response of clean Ag-coated $\mathrm{Nb}$ proximity cylinders in the $\mu \mathrm{K}$ region. In the mesoscopic temperature regime the normal-metal-superconductor system shows the as yet unexplained paramagnetic reentrant effect, discovered some years ago [38]. The theoretical paper [39] reported numerical results for the local density of states in the semiclassical Andreev billiards. It was shown that an energy gap develops in a chaotic billiards near the Fermi energy, but there is no gap found in a circular billiards.

For our system we believe that the contact between the pure normal and superconducting metals is good. As a result, Andreev scattering [40] of 
quasiparticles appears to be the main mechanism responsible for the formation of quantum states in the normal metal. It is assumed that the width of the superconducting layer on the cylinder boundary is of the order of the coherence length $\xi_{0}$. In this case the fluxoid is quantized and the value of the trapped flux may change continuously. It is assumed that the cylinder radii $R>>\xi_{0}$.

A dispersion relation has been derived for the excitation spectrum, and the quasiclassical picture of the quasiparticle motion has been analyzed.

In previous studies on normal cylinders $[7,8]$ the formation of quantum states was associated with specular electron reflection at the metal boundary. After each reflection from the boundary, the electron traveled along a trajectory tangent to a caustic whose radius was dependent on the magnetic quantum number. After Andreev scattering at the NS boundary of the cylinder, the trajectory of the quasiparticles is more complicated. The energy of a «particle» type of excitation is higher than the Fermi energy, and its momentum exceeds the Fermi momentum $\hbar k_{F}$, too. As a result of Andreev scattering the «particle» transforms into a «hole» whose momentum is smaller than $\hbar k_{F}$. It follows from the law of conservation of angular momentum that the «particle» and the «hole» have different caustic radii, the caustic radius of the latter being larger. This feature of the quasiparticle motion was taken into account in deriving the condition for quasiclassical quantization of excitations. That condition for quantization has been obtained within the multidimensional quasiclassical method, which permit us to determine the spectrum of the Andreev levels and to calculate the density of states of the system in question.

The density of states as a function of $\Phi$ has a steplike behavior and experiences jumps at $\Phi=h c(s+1) / 2 e$ (where $s$ is a natural number), i.e., it has a resonance character. The distance between steps is equal to the flux quantum $h c / 2 e$. Giant-amplitude oscillations of the conductance of an SNS contact were predicted in [41,42] for the case when the phase difference of the order parameter of the superconductors is equal to an odd number times $\pi$. For our system the resonance spikes of the density of states can be explained as follows.

Let us consider two points $A$ and $B$ on a given section of cylinder. Let us assume that a quasiparticle moves along the line $A B$. In the case where the distance $A B$ is of a mesoscopic scale there exist a Josephson current between these points. The energy spectrum of such a local SNS contact is determined by the order parameter phase difference at points $A$ and $B$, which depends on the flux $\Phi$ and the radial angle $\alpha$ between those points. The energy of the Andreev level reaches the Fermi surface whenever $\Phi=\Phi_{0}(s+1)$. In this case the spectrum exhibits strong degeneracy insofar as it becomes independent of the longitudinal (along the cylinder axis) component of the quasimomenta. The range of degeneracy depends on the total flux $\Phi$. Therefore, in this case the density of states experiences a resonance increase with amplitude, showing a steplike behavior with increasing $\Phi$.

\section{Formulation of the problem}

Let us consider a pure simply connected normal cylinder of radius $R$. The particles with energy $E<\Delta$ experience Andreev scattering at their superconducting boundaries. It is assumed that the width of the superconducting layer is of the order of the coherence length $\xi_{0}\left(\right.$ or $\lambda_{L}$ ). In this case fluxoid $\Phi_{L}$ is quantized,

$$
\Phi_{L}=\Phi+\frac{\pi \lambda_{L}^{2}}{c} \oint \mathbf{j} d \mathbf{s}=n \Phi_{0}, n=0,1,2, \ldots,
$$

and the value of the trapped magnetic flux $\Phi=\eta \Phi_{0}$ through the superconductor cross section may change continuously ( $\lambda_{L}$ is the London penetration depth, $j$ is the superfluid current, and $\Phi_{0}=h c / 2 e$.

In this Section we deduce a quasiclassical equation describing the spectrum of quasiparticles in the normal part of the cylinder. To clear up the systematics of the states it is useful to proceed from the Bogoliubov-de Gennes equations [43]:

$$
\begin{gathered}
\hat{H}_{e} u(\mathbf{r})+\Delta(\mathbf{r}) v(\mathbf{r})=E u(\mathbf{r}), \\
-\hat{H}_{e}^{*} v(\mathbf{r})+\Delta^{*}(\mathbf{r}) u(\mathbf{r})=E v(\mathbf{r}) .
\end{gathered}
$$

Here $\left(\begin{array}{l}u \\ v\end{array}\right)$ is the two-component wave function of a quasiparticle, $E$ stands for the energy eigenvalues,

$$
\hat{H}_{e}=\frac{1}{2 m^{*}}\left[-i \hbar \nabla-\frac{e}{c} \mathbf{A}\right]^{2}-\zeta
$$

is the single-particle Hamiltonian dependent on the vector potential $\mathbf{A}, \zeta$ is the chemical potential of the metal, and $m^{*}$ is the effective electron mass. It is hard to solve Eq. (1) exactly, but the problem can be much simpler if we use the stepwise potential approximation for $\Delta$. Within the gauge chosen for 
A, the order parameter is preassigned by the expression:

$$
\begin{gathered}
\Delta(\mathbf{r})=\Delta(r) \exp (-i[\eta] \theta), \\
\Delta(r)= \begin{cases}\Delta, & r>R, \\
0, & r<R,\end{cases}
\end{gathered}
$$

where $\theta$ is the angle, which varies in the interval $0 \leq \theta \leq 2 \pi$, and $[\eta]$ is the integer part of $\eta$. With this choice of the pairing potential it is a single-valued function of the coordinates. We disregard the distortions of the excitation trajectories caused by the magnetic field. In this study we confine our consideration to the vector potential field, taking into account its influence on the phase increment in the wave function of the excitation. The case of weak magnetic field will be the subject of another paper.

As was shown in Refs. 7 and 8, the interference patterns of the oscillations of thermodynamic values have a common cause, namely the Aharonov-Bohm effect. To describe the field of the vector potential, we shall consider a normal hollow cylinder whose inner radius tends to zero. Let us assume that a solenoid is inserted into the orifice of the cylinder. It excites the field of the vector potential. It is hard to realize the situation in practice. Nevertheless, it is interesting to consider it because it demonstrates unambiguously the fact of flux quantization. According to a calculation [8], in a simply connected cylinder the oscillations that arise have the largest amplitudes in the vector potential field because they take contributions from all electron states with arbitrary magnetic quantum numbers. When such a cylinder, with specular reflection of quasiparticles at the dielectric boundary, is placed in a weak magnetic field, the surface states with the highest magnetic quantum numbers $m$ (the «whispering gallery» type states) are responsible for the oscillations with the quantum flux period $h c / e$ [8].

Having performed the gauge transformation in Eqs. (1), we can change over to the real pairing potential. In this case the wave function can be written as

$$
\begin{aligned}
& u(\mathbf{r})=u^{\prime}(\mathbf{r}) \exp \left(-i \frac{[\eta]}{2} \theta\right) \\
& v(\mathbf{r})=v^{\prime}(\mathbf{r}) \exp \left(i \frac{[\eta]}{2} \theta\right)
\end{aligned}
$$

The components $u^{\prime}$ and $v^{\prime}$ of the new wave function are dependent only on the radial variable. Therefore, Eqs. (1) can be solved as

$$
\begin{aligned}
& u^{\prime}(\mathbf{r})=u^{\prime}(r) \exp [i(m \theta+q z)], \\
& v^{\prime}(\mathbf{r})=v^{\prime}(r) \exp [i(m \theta+q z)],
\end{aligned}
$$

where $m$ is the magnetic quantum number, and $q$ is the wave vector component along the axis of the cylinder. Proceeding from the single-valuedness requirement for the initial wave functions, we arrive at the following condition: the parity of $2 \mathrm{~m}$ is determined by the parity of the trapped flux quanta

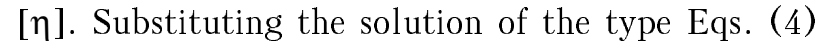
into Eqs. (1), we obtain the equation for the radial function of the problem. Its matrix form is

$$
\begin{gathered}
\sigma_{z} \frac{\hbar^{2}}{2 m^{*}}\left[-\frac{d^{2}}{d r^{2}}-\frac{1}{r} \frac{d}{d r}+\left(m-\sigma_{z} \frac{\tilde{\eta}}{2}\right)^{2} \frac{1}{r^{2}}-\right. \\
\left.-\left(k_{F}^{2}-q^{2}\right)\right] R_{ \pm}(r)+\sigma_{x} \Delta(r) R_{ \pm}=E R_{ \pm}(r)
\end{gathered}
$$

where $\sigma_{x}, \sigma_{z}$ are the Pauli matrices, $\tilde{\eta}=\eta+[\eta]$, and the \pm on $R$ refers to a «particle» or a «hole» radial function, respectively. In a normal metal $(r<R) \Delta(r)=0$, and Eq. (5) reduces to the equation for the Bessel function:

$$
R(r) \sim J_{m \tilde{\mp} / 2}\left[\left(k_{r} \pm \frac{E}{\hbar v_{r}}\right) r\right] .
$$

Here the notations $k_{r}=\sqrt{k_{F}^{2}-q^{2}}$ (the radial wave number) and $v_{r}$ (the radial velocity) are introduced. The solution of Eq. (6) for $\eta=1$ coincides in form with the wave function found for the vortex lines of a pure type II superconductor [44,45], if magnetic effects are neglected. We can thus see that the angular momentum is $\hbar(m-\tilde{\eta} / 2)$ for the «particle» and $\hbar(m+\tilde{\eta} / 2)$ for the «hole».

In the superconducting region $(r>R)$ the solution of Eq. (5) is expressed in terms of the Hankel functions $H_{m}^{(1)}\left(k_{r} r\right)$ and $H_{m}^{(2)}\left(k_{r} r\right)$. Matching these solutions at the NS boundary, we can obtain the equation for the quasiparticle spectrum. It is, however, hard to derive the general expression for the quasiparticle spectrum of the system by this method even in the case of our chosen stepwise potential $\Delta(r)$. The thermodynamic values can be calculated more conveniently using the approach based on derivation of the equation for the spectrum which gives asymptotically correct estimates of the energy levels. To do this we employ the multidimensional quasiclassical method.

Keller and Rubinow [46] have generalized the conditions of the Bohr-Sommerfeld quasiclassical quantization. Commonly, such conditions have been 
derived assuming that the quasiclassical wave function was

$$
\Psi_{0}\left(q_{i}, t\right)=A\left(q_{i}, t\right) \exp \left(i S\left(q_{i}, t\right) / \hbar\right),
$$

where $S\left(q_{i}, t\right)$ is the total action and the amplitude $A\left(q_{i}, t\right)$ is a single-valued function of the coordinates $q_{i}$. Because of continuity of the function $\Psi_{0}$, the difference $\Delta S$ between any two values of the multiple-valued function $S\left(q_{i}, t\right)$ is

$$
\Delta S=\oint_{\mathcal{L}} p_{i} d q_{i}=2 \pi \hbar n, n=0,1,2, \ldots
$$

This equation should be valid for an arbitrary closed curve in $q$ space. The integral in Eq. (8) is the same for any closed curves which can be transformed one into another without crossing the singularity of the integrand (equivalent contours). The authors of [46] assume that the amplitude $A\left(q_{i}, t\right)$ is also a multiple-valued function of the coordinates, and they write

$$
\Psi_{0}=\sum_{k} A_{k}(q, t) \exp \left(\frac{i S_{k}(q, t)}{\hbar}\right)
$$

instead of Eq. (7). Here $S_{k}$ and $A_{k}$ are the branches of the multiple-valued functions $S$ and $A$. Then the requirement of continuity of $\Psi_{0}$ leads to

$$
\Delta S=\oint_{\mathcal{L}} \nabla S_{k} d \mathbf{s}=2 \pi \hbar\left(n+\frac{i}{\pi} \oint_{\mathcal{L}} \nabla \ln A_{k} d \mathbf{s}\right),
$$

where $d \mathbf{s}$ is the length element along the path $\mathcal{L}$.

Since the functions $\nabla S$ and $\nabla \ln A$ are multiplevalued (e.g. , $M$-multiple), we can join $M$ sheets of our $q$ space and introduce the notion of a covering space in which the functions $S$ and $\ln A$ become single-valued. The independent (nonequivalent) contours in the covering space give the number of quantization conditions [46,47].

The increment in the value $\underset{\mathcal{L}}{\oint} \nabla \ln A d \mathbf{s}$ in Eq. (10) should be calculated taking into account the fact that the function $\ln A$ changes at the contour points where $A$ goes to $\infty$. This occurs on caustic surfaces and surfaces of the points at which the quasiparticle stops (i.e., its velocity becomes zero). Keller and Rubinow [46] obtained the generalized quantization conditions taking into account the contributions from the above specific points in the contour $\mathcal{L}$ :

$$
\oint_{\mathcal{L}} p_{i} d q_{i}=2 \pi \hbar\left(n+\frac{d}{4}+\frac{b}{4}\right)
$$

( $d$ is the number of intercepts of the contour $\mathcal{L}$ and the caustic surfaces, and $b$ is the number of stop points). The quantization conditions of Eq. (11) are valid both for the smooth potentials in the Schrödinger equation and for the free motion of a particle in a region confined within hard walls that reflect the particle specularly.

\section{$A$ rigid-wall circle}

Keller and Rubinow's method permits construction of asymptotic expressions for the exact wave functions and the eigenvalues of the problem. Here we briefly outline the method for a particular example. Let a particle move freely in a circular region with the radius $R$ restricted by rigid walls. Its behavior can be described by the Schrödinger equation

$$
\left(\Delta+k^{2}\right) \Psi=0, \quad k^{2}=\frac{2 m^{*} E}{\hbar^{2}} .
$$

It is required that $\Psi$ be zero at the boundary. The quasiclassical solution is searched for assuming that for high $k$ values the wave function has the form

$$
\Psi=\sum_{j=1}^{N} \mathrm{e}^{i k S_{j}}\left[A_{j}+O\left(\frac{1}{k}\right)\right] .
$$

Let us assume further that each term in Eq. (13) complies with Eq. (12). Substituting Eq. (13) into Eq. (12) and setting the coefficients of $k^{2}$ and $k$ equal to zero, we can obtain the equations for $S_{j}$ and $A_{j}$ with any arbitrary $j$ [46]:

$$
\left\{\begin{array}{l}
(\nabla S)^{2}=1, \\
2 \nabla S \nabla A+A \Delta S=0 .
\end{array}\right.
$$

The first of Eqs. (14) is the eikonal equation for geometric optics. The other has the meaning of the law of conservation of «probability» for $|A|^{2}$. $S=$ const can be interpreted as the wave-front surface. The rays orthogonal to these surfaces are straight lines. To fulfill the boundary condition $\Psi=0$, the terms should enter Eq. (13) in pairs satisfying the conditions:

$$
S_{j}=S_{j^{\prime}}, \quad A_{j}=-A_{j^{\prime}}, \quad \frac{\partial S_{j}}{\partial n}=\frac{\partial S_{j^{\prime}}}{\partial n} .
$$

In passing through the caustics the phase with the amplitude $A$ changes by $-\pi / 2$, and on reflection 
at the boundary it changes by $-\pi$ (see Eq. (15)). The quantization condition takes the form [46]

$$
\oint_{\mathcal{L}} p_{i} d q_{i}=2 \pi \hbar\left(n+\frac{d}{4}+\frac{b}{2}\right)
$$

To solve the eigenvalue problem, it is necessary to consider all possible rays inside the circular region. These rays are tangent to the circle of radius $a_{0}$ which acts as a caustic for them. In this case two families of rays pass through each point of the ring, and each family occupies completely the region inside the circle (Fig. 1). On each event of reflection at the boundary, the rays change over from one family to the other. It is easily seen that in this case the covering space consists of two circular rings which are joined along their peripheries, their radii being $R$ and $a_{0}$. Topologically, the covering space is a torus. The torus has only two independent contours which do not contract to a point. Consequently, the number of quantization requirements necessary to describe the motion of the wave inside the circular region reduces to two. Let us choose the circumference with the caustic radius $a_{0}$ as one of the integration contours. It does not cross the caustic, and therefore $d=b=0$. On the basis of Eq.(16) this gives the condition of angular momentum quantization:

$$
k 2 \pi a_{0}=2 \pi m, m=0,1,2, \ldots
$$

The other contour on the toroidal surface can be deformed (without affecting the integral) as shown in Fig. 2. Calculating the contour integral, in Eq. (16) and taking into account that $d=b=1$, we can obtain, by means of Eq. (17), a transcendental equation for the eigenvalues of the wave vector [46]:

$$
\begin{gathered}
\sqrt{k^{2} R^{2}-m^{2}}-m \arccos \frac{m}{k R}=\pi\left(n+\frac{3}{4}\right), \\
n, m=0,1, \ldots
\end{gathered}
$$

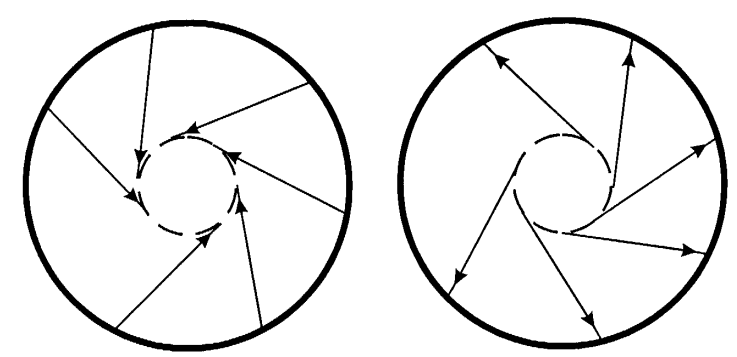

Fig. 1. Two congruences of rays in a circular domain of the cylinder. The concentric circle of radius $a_{0}$ is the caustic of these rays.

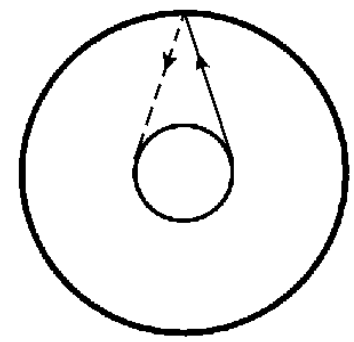

Fig. 2. A closed curve on the toroidal covering space associated with two ray congruences, considered in Fig. 1.

This method also permits us to find an analytical expression for the wave function. Keller and Rubinow compared it with the exact solution of the problem,

$$
\Psi \sim J_{m}(k R) \mathrm{e}^{i m \theta},
$$

and thus showed that the quasiclassical solutions obtained for eigenfunctions and the eigenvalues are asymptotically exact. In the region between the caustics and the sample boundary, the Debye asymptotics of the Bessel function $(1<<m<k R)$ is used as an asymptotic approximation to the function $J_{m}(k R)$. The solution attenuates exponentially inside the caustic $(m>k R)$.

\section{SNS contact in zero magnetic field}

We can show that similar quasiclassical quantization conditions can be written for structures in which excitations are reflected at the boundary of their localization region by the Andreev mechanism. Let us first consider an SNS contact with flat parallel NS boundaries. We analyze a family of parallel rays (trajectories) directed at a certain angle $\alpha$ to the normal to the film boundary. We choose one of the rays as a path of integration $\mathcal{L}_{i}$. The covering space can be constructed out of two similar $q$ spaces (of SNS contacts) in which the quasiparticles move in opposite directions. On each event of the Andreev reflection the amplitude of the wave function of quasiparticle is multiplied by the factor

$$
\gamma=\mathrm{e}^{-i \arccos E / \Delta} .
$$

For a closed contour $\mathcal{L}_{i}$ the complete change of the phase on the NS boundaries is:

$$
\frac{I}{2 \pi}(-i) 2 \arccos E / \Delta=\frac{1}{\pi} \arccos E / \Delta .
$$


In the case of a current-carrying state, the difference between the superconducting order parameter phases $\varphi$ of two the given superconductors is added to the phase Eq. (21). Assuming $d=b=0$, we obtain the generalization of the Eq. (11) for a SNS contact:

$$
\oint_{\mathcal{L}} p_{i} d q_{i}=2 \pi \hbar\left(n+\frac{1}{\pi} \arccos \frac{E}{\Delta} \pm \frac{\varphi}{2 \pi}\right) .
$$

Let us denote the quasiparticle momentum inside the $\mathrm{N}$ layer as $K$ :

$$
K= \begin{cases}p_{0}=\sqrt{2 m^{*}(\tilde{\zeta}+E)}, & \text { «particle»; } \\ p_{1}=\sqrt{2 m^{*}(\tilde{\zeta}-E)}, & \text { «hole», }\end{cases}
$$

where $\tilde{\zeta}$ is the chemical potential of the metal minus the energy of the motion along the NS boundary. Proceeding from Eq. (22), we obtain

$$
\begin{gathered}
\oint_{\mathcal{L}} K d \mathbf{s}=\int p_{0} d s-\int p_{1} d s= \\
=2 \pi \hbar\left\{n+\frac{1}{\pi} \arccos \frac{E}{\Delta} \pm \frac{\varphi}{2 \pi}\right\} .
\end{gathered}
$$

Introduction of the quasiparticle velocity along the path of the motion leads to the expression for the spectrum of the SNS contact which was obtained by Kulik [48]

$$
E_{n}=\frac{\pi \hbar v_{F}}{\mathcal{L}}\left\{n+\frac{1}{\pi} \arccos \frac{E}{\Delta} \pm \frac{\varphi}{2 \pi}\right\} .
$$

\section{Andreev reflection of excitations at the cylinder boundaries}

Let us deduce an equation for the spectrum of quasiparticles with energies $E<\Delta$ which move inside a normal cylindrical conductor with a superconducting coating. The normal metal is assumed to be pure. Excitations move in it ballistically and experience Andreev scattering at the NS boundary. We shall analyze the features of the quasiparticle trajectories within the system studied. Let us assume that the normal metal initially has no trapped magnetic flux, $\Phi=0$. The tangential components of the wave vector of the quasiparticle are conserved at the NS boundary of the metal during the scattering of a «particle» into a «hole» (and vice versa). The angular momentum of the «particle» and «hole» are conserved, too. Since the «hole» has a smaller quasimomentum than the «particle», the caustic radius $a_{1}$ of the hole should be larger than the caustic radius $a_{0}$ of the particle. Therefore the angles of incidence of the «particle» and the «hole» at the NS boundary will be different, that of the hole being smaller. Thus the quasiparticle trajectory is not strictly recursive. When a certain flux $\Phi$ is trapped in the normal cylinder, the angular momentum of the «hole» increases to $\hbar(m+\tilde{\eta} / 2)$, while that of the «particle» decreases to $\hbar(m-\tilde{\eta} / 2)$. This implies that at $\Phi \neq 0$ the difference between the angles at which the «particle» and the «hole» approach the boundary becomes larger. The change in the angular momentum in the case of $\Phi \neq 0$ is caused by the screening superfluid current in the $\mathrm{S}$ layer of the structure. The qualitative form of the quasiparticle trajectory inside the cylinder cross section is shown in Fig. 3.

The multidimensional quasiclassical method can be generalized readily to the motion of quasiparticles experiencing Andreev scattering at the boundaries of a circular region. Instead of Eqs. (14), we obtain similar separate equations for «particles» and for «holes». The wave function $\left(\begin{array}{l}u \\ v\end{array}\right)$ of the initial equation (1) in the preassigned field of the vector potential A ( $\left.A_{r}=A_{z}=0 ; A_{\theta}=\Phi / 2 \pi r\right)$ is a singlevalued function of $\mathbf{r}$ on a complete circuit over the cylinder surface. After the gradient transformation $\mathbf{A}^{\prime}=\mathbf{A}+\nabla \chi$ using the function $\chi=+\hbar c[\eta] \theta / 2 e$, the pairing potential $\Delta$ becomes real and the new wave function $\left(\begin{array}{l}u^{\prime} \\ v^{\prime}\end{array}\right)$ differs from the former $\left(\begin{array}{l}u \\ v\end{array}\right)$ in having a factor $(-1)^{[\eta]}$, where $[\eta]$ is the number of quanta of the trapped flux [43]. The phase increment in the

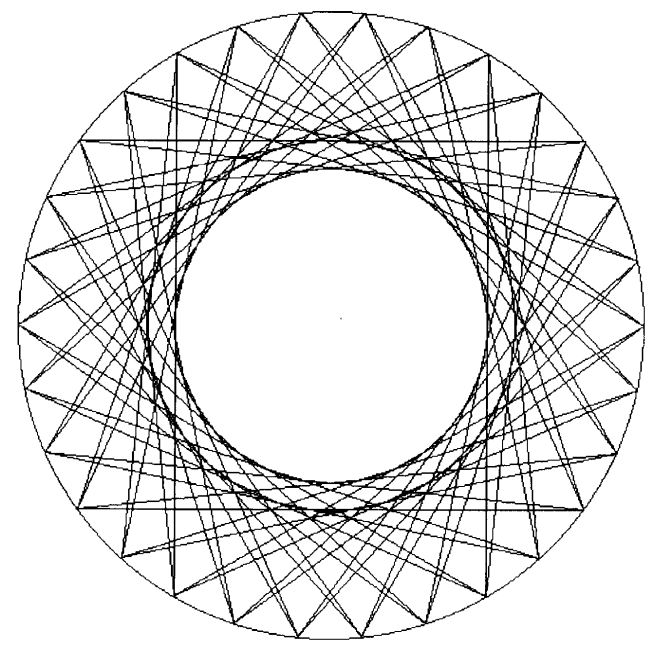

Fig. 3. Quasiclassical picture of the quasiparticle trajectories inside the circular normal domain of the metal coated with a superconducting layer. The «particle» and «hole» trajectories are tangents to caustics of different radii. 
wave function of the «particle» on the circular contour of the radius $a_{0}$ is $\oint\left(k_{0}+\frac{e}{\hbar c} \mathbf{A}^{\prime}\right) d \mathbf{s}=2 \pi m$. The phase increment of the wave function of a «hole» can be found similarly on the contour of radius $a_{1}$. As a result, we arrive at the expressions

$$
k_{0} a_{0}=m-\tilde{\eta} / 2, \quad k_{1} a_{1}=m+\tilde{\eta} / 2 .
$$

The derivation of the second equation in (26) takes into account the fact that the effective charge of the «hole» is opposite in sign to the charge of the «particle».

The problem of the energy eigenvalues can be solved using a geometrical approach. As seen in Fig. 3 , the ray tangential to the caustic radius $a_{0}$ describes the motion of a «particle». The ray of a «hole» is tangent to the caustic radius $a_{1}$. Let us first consider the rays describing the motion of the «particle». As in the case of specular reflection from the boundary, the circular ring between the radii $R$ and $a_{0}$ contains two families of such rays. Family I includes the rays moving from the NS boundary to the caustic $a_{0}$, and family II consists of the rays moving in the opposite direction. At the NS boundary the rays of family I transform into those of family II. After Andreev scattering on the off-diagonal potential, a «particle» transforms into a «hole», for which we can also introduce two families of rays moving inside the circular ring and having the radii $R$ and $a_{1}$. Thus, in our case the covering space consists of two circular rings for «particles» joined along the circumferences of radii $R$ and $a_{0}$ and two rings for «holes» joined along the circumferences of radii $R$ and $a_{1}$. Topologically, this space has the form of two tori, one inside the other, whose surfaces have a common contact line along the radius $R$. Such surface has only three independent contours which do not contract to a point. Two of them give quantization conditions relating the corresponding angular momentum, quasimomenta, and caustic radii of the «particle» and the «hole» (see Eq. (26)).

The third contour on the covering surface can be deformed to the shape shown in Fig. 4. The contour integral can be calculated taking into account the condition $\varphi=0$ and the increment in the wave function phase at each Andreev scattering event. Proceeding from Eq. (26), we thus obtain the equation for the spectrum of quasiparticles inside the cylinder:

$$
\sqrt{k_{0}^{2} R^{2}-m_{0}^{2}}-\sqrt{k_{1}^{2} R^{2}-m_{1}^{2}}-m_{0} \arccos \frac{m_{0}}{k_{0} R}+
$$

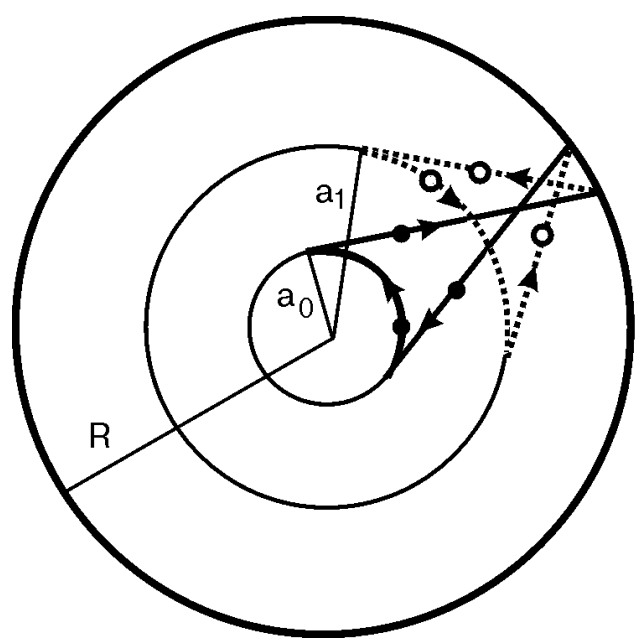

Fig. 4. Shape of the integration path used to derive a quasiclassical equation for the spectrum of Andreev levels. For illustration, two intersection points between the integration path and the NS boundary are mutually shifted so that they could show the processes of «particle»-to-«hole» and backward scattering.

$$
+m_{1} \arccos \frac{m_{1}}{k_{1} R}=\pi\left(n+\frac{1}{2}+\frac{1}{\pi} \arccos \frac{E}{\Delta}\right) .
$$

Here $k_{0}$ and $k_{1}$ are the wave vectors of a «particle» and a «hole», respectively, in the $\mathrm{N}$ layer (their expressions are given in Eq. (23)); $\hbar m_{0}=\hbar(m-\tilde{\eta} / 2)$ and $\hbar m_{1}=\hbar(m+\tilde{\eta} / 2)$ are their angular momenta, $m$ is the absolute value of the magnetic quantum number, and $n=0,1,2 \ldots$ The quasiclassical parameter of the problem is $k_{0} R \cong k_{1} R \cong k_{F} R>>1$.

Note that the coefficient $1 / 2$ within the brackets on the right-hand side of Eq. (27) shows the change in the wave function phase during the quasiparticle scattering at the caustics $a_{0}$ and $a_{1}$. The number of intersections of the path of integration and the caustic surface is two, as is seen in Fig. 4. We should then put $d=2$ and $b=0$ in Eq. (16) and take into account that because of the two Andreev reflections of the quasiparticle at the NS boundary, the phase has the increment $-\arccos E / \Delta$ (see Eq. (21)).

As follows from Eq. (27) the energy $\varepsilon$ in units of $\hbar^{2} / 2 m^{*} R^{2}$ and the quantum numbers $m, q$ are associated by the condition: $\left(k_{F}^{2}-q^{2}\right) R^{2} \geq \varepsilon+m_{1}^{2}$. In order to clarify its meaning we introduce the angle $\vartheta$ at which the quasiparticle is incident on the NS boundary: $\sqrt{k_{F}^{2}-q^{2}}=k_{F} \sin \vartheta$. In this case, for a fixed $m$ the minimal angle of incidence for the quasiparticle with energy $\varepsilon$ is equal to $\vartheta_{\min }=\arcsin \left(\sqrt{\varepsilon+m_{1}^{2}} / k_{F} R\right)$. Assuming $m_{1} \approx 0$, we see that the limiting value of the angle is $\vartheta \sim \sqrt{E / \zeta}$, below which a change of the Andreev 
reflection on the conventional specular reflection on the off-diagonal potential of the superconductor takes place $[49,50]$.

Equation (27) implicitly determines the dependence of the $E$ levels on the quantum numbers $n, m$, $\hbar q: E_{n m}(q)$.

Let us consider special cases of Eq. (27).

i) Let $m \approx 1, \eta \approx 0$. A quasiparticle moves along the cylinder diameter. When the radius $R$ is large, the situation resembles an SNS contact. Indeed, in this case Eq. (27) can give a spectrum resembling that obtained by Kulik, where the phase difference of the order parameters is zero,

$$
E_{n}=\frac{\pi \hbar v_{r}}{2 R}\left(n+\frac{1}{2}+\frac{1}{\pi} \arccos \frac{E}{\Delta}\right),
$$

where $v_{r}=\sqrt{2 \tilde{\zeta} / m^{*}}$.

ii) Let the trapped flux be $[\eta]=1$. The magnetic quantum number $2 m$ is then half-integer. Expanding Eq. (27) into a power series of the small parameter $1 / k_{F} R<<1$ gives

$$
E=\frac{\Delta m}{k_{F} R} .
$$

If $R \approx \xi_{0}$ ( $\xi_{0}$ is the coherence length), the spectrum in Eq. (29) coincides with the spectrum of lowlying levels of the vortex lines of the type II superconductor $[44,45,52]$, if all magnetic effects are neglected:

$$
E=\frac{\pi \Delta^{2} m}{2 \zeta}
$$

Finally, note the following particularity of the system, seen from the Eq. (27). In the limiting case $k_{F} R>>1$, after expanding the left-hand side of Eq. (27) up to first order in $1 / k_{F} R$ and taking the limit $\varepsilon \rightarrow 0$ one obtains $\pi \tilde{\eta} / 2-\tilde{\eta} m / k_{F} R=\pi(n+$ $+1)$. Thus to zeroth order in $1 / k_{F} R$

$$
\tilde{\eta}=2(n+1) \text {. }
$$

We will show below that whenever the trapped flux $\Phi$ in the N layer is a multiple of the flux quantum $h c / 2 e$, the mesoscopic system in question manifests resonance.

\section{Density of states of normal excitations in a cylinder}

Let us calculate the density of states $v(E)$ for excitations localized in a normal cylinder which experience Andreev scattering at the NS boundary. We proceed from

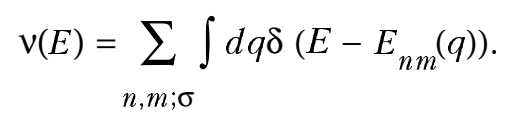

The summation is over all quantum numbers and the spin $\sigma$.

Equation (27) determines the quasiparticle spec$\operatorname{trum} E_{n m}(q)$. In the quasiclassical approximation an analytical solution could be obtained. In particular, expanding the left-hand side of the Eq. (27) in $1 / k_{F} R<1$ and taking into account Eq. (23), we obtain

$$
E_{n}(q)=\frac{\pi \hbar v_{r}(q)}{2 R|\sin \alpha / 2|}\left(n-\frac{\tilde{\eta}}{2 \pi} \alpha+\frac{1}{2}+\frac{1}{\pi} \arccos \frac{E}{\Delta}\right) .
$$

The spectrum (32) is similar to that of the SNS contact obtained by Kulik [48]. This spectrum corresponds to motion of quasiparticles between two points on the cylinder surface parametrized by the radial angle $\alpha$, while the distance between these points is $2 R|\sin \alpha / 2|$. However, contrary to the case of a standard SNS contact the phase difference $-\tilde{\eta} \alpha / 2 \pi$ depends on the flux. For given $\alpha$, the spectrum (32) describes an «SNS» contact with an effective density of states $v(E ; \alpha)$. The total density of states is given by:

$$
v(E) \sim \int_{0}^{2 \pi} v(E ; \alpha) d \alpha .
$$

The spectrum (32) was obtained on the assumption that the quasiparticle energy $E$ was close to the Fermi energy $\zeta$. As was noted in the Introduction, the Andreev levels of the mesoscopic system can shift when the flux changes; at a certain value of the flux they can coincide with $\zeta$. For low energies $E$, the quasimomenta of a «particle» and a «hole» (and the radii $a_{0}$ and $a_{1}$ of their caustics) become practically identical. We may therefore assume that in the description of these two states the quasiclassical trajectories would be simplier in shape than in Fig. 3: when the «particle» is reflected from the NS boundary, the «hole» comes back to its initial point at the cylinder surface. Within our approximation (32), we should take into account that inside the cylinder cross section there exist a great number of «SNS contacts» with the preassigned chord length, i.e., these states possess high degenerate multiplic- 
ity about $N \sim 2 \pi R / \lambda_{B}>>1\left(\lambda_{B}\right.$ is the de Broglie wavelength). We took this fact into consideration when calculating the density of states of the system in the vicinity of resonance.

As a matter of fact, calculation of the thermodynamic quantities far off resonance should be based on the general equation for the spectrum of quasiparticles, Eq. (27).

The resonance contribution to $v(E)$ can be calculated using the spectrum of lower energy levels

$$
E_{n}(q)=\frac{\pi \hbar v_{r}(q)}{2 R|\sin \alpha / 2|}\left(n+1-\frac{\tilde{\eta}}{2 \pi} \alpha\right) .
$$

On substituting Eq. (34) into Eq. (31), integrating over $q$ and $\alpha$, and summing over spin, we obtain

$$
\nu(\varepsilon) \sim A \varepsilon \int_{0}^{\pi} d \alpha \times
$$

$$
\sum_{n=-\infty}^{+\infty} \frac{\sin ^{2} \frac{\alpha}{2} \theta\left(n+1-\frac{\tilde{\eta}}{2 \pi} \alpha-\varepsilon \sin \frac{\alpha}{2}\right)}{\left(n+1-\frac{\tilde{\eta}}{2 \pi} \alpha\right)\left[\left(n+1-\frac{\tilde{\eta}}{2 \pi} \alpha\right)^{2}-\varepsilon^{2} \sin ^{2} \frac{\alpha}{2}\right]^{1 / 2}},
$$

where

$$
A=\frac{8 L R m^{*}}{\pi^{2} \hbar^{2}} \frac{2 \pi R}{\lambda_{B}}
$$

$L$ is the cylinder height, and $\theta(x)$ is the step function, equal to unity at $x>0$ and to zero at $x<0$. We introduce the dimensionless energy $\varepsilon=E / E_{0}, E_{0}=\hbar^{2} /\left(2 m^{*} R^{2}\right)$. As Eq. (35) shows, for a preassigned flux, the denominator of the radicand can become zero at a certain angle $\alpha=\alpha(\Phi)$. When $\varepsilon \rightarrow 0$ one has $\alpha_{n} \simeq(n+1) 2 \pi / \tilde{\eta}$. In this case the inequalities

$$
0 \lesssim \alpha_{n} \lesssim \frac{(n+1) 2 \pi}{\tilde{\eta}} \lesssim \pi ; \eta=\frac{\Phi}{\Phi_{0}}
$$

are fulfilled, which leads to the condition $\eta+[\eta] \gtrsim 2(n+1)$. Thus, with a preassigned flux $\Phi$, the number of terms in the series of Eq. (35) is limited, and it increases with growing $\Phi$.

Prior to calculation of $v(\varepsilon)$, let us discuss the question of the contributions of different angles $\alpha_{n}$ to the resonance amplitude. It is reasonable to assume that because of the factor $\sin ^{2} \alpha / 2$ in the numerator of Eq. (35), the angles $\alpha_{n} \approx \pi$ are the main contributors to the integral. For such an $\ll$ SNS contact», the Josephson current flows through the cross section of the cylinder along its diameter. On the other hand, since the resonance condition (35) contains the product of the flux times the angle $\alpha$, we may expect an extra contribution to the amplitude of the effect from smaller angles $\alpha_{n}$ when the flux increases. As a matter of fact, this expectation is based on the approximate resonance condition obtained from Eq. (35) in which the first term in the radicand is kept while the second term, $\varepsilon^{2} \sin ^{2} \alpha / 2$, is dropped.

We thus arrive at the conclusion that the main contribution to the resonance of the density of states appears near the angle $\alpha=\pi$. Introducing the notation $\xi=\pi-\alpha<<1, \quad a=n+1-\tilde{\eta} / 2, \quad b=$ $=\tilde{\eta} / 2 \pi$, we obtain the equation for the resonance condition:

$$
a^{2}+b^{2} \xi^{2}+2 a b \xi-\varepsilon^{2}\left(1-\frac{\xi^{2}}{4}\right)=0,
$$

whose solution is $\xi \simeq-a / b \pm \varepsilon$. It is seen that for $b \sim 1$, the energy $\varepsilon$ and the value of $a$ are both small, but the condition $a \geq \varepsilon$ is always fulfilled. The expression in brackets in front of the radical in Eq. (35) is therefore of the order of the energy $\varepsilon$ and cancels with the energy factor of $\varepsilon$ in front of the integral sign. The remaining integral is estimated to be a constant of about unity.

The resonance-induced spike of the density of states always appears when the Andreev level coincides with the Fermi energy at a certain flux in the $\mathrm{N}$ layer. The sharp increase in the amplitude $v(E)$ at $E \rightarrow 0$ is caused by the integral contribution of the states of quasiparticles indexed by the quantum number $q$, which describe the quasiparticle motion along the sample axis.

When the resonance is disturbed, the condition $(n+1-\tilde{\eta} \alpha / 2 \pi) \neq 0$ is fulfilled, and for low energies $\varepsilon \rightarrow 0$ we find

$$
v^{(0)}(\varepsilon) \sim 2 \pi^{3} \varepsilon \int_{0}^{1} \frac{d x \sin ^{2} \pi x}{\cos ^{2}\left[2 \pi^{2} b x\right]} \sim \varepsilon .
$$

Near the resonance, the ratio of the resonance and nonresonance amplitudes of the density of states is 


$$
\frac{v^{\text {res }}}{v^{(0)}} \sim \frac{1}{\varepsilon}>>1
$$

It is thus shown that on variation of the trapped magnetic flux, the density of states of a normal cylindric conductor coated with a thin superconducting layer $\left(\sim \xi_{0}\right)$ is described by a stepped function $\Phi$. The step spacing is equal to a superconducting flux quantum $\Phi_{0}$ and the step height increases with the flux. Differentiation of the density of states with respect to $\Phi$ gives a set of resonance spikes spaced at $h c / 2 e$.

The physical reason for the features having a period of the superconducting flux quantum $\Phi_{0}$ is as follows. As seen in the spectrum of Eq. (27), for the preassigned magnetic flux $\Phi, q$, and the energy, there is a highest magnetic quantum number $m$ which can be used to describe the Andreev levels. This quantum number is responsible for the largest caustic realizable for quasiparticles moving ballistically near the cylinder boundary. At a certain value of the changing flux, the caustic «crawls» through the NS boundary of the cylinder, an event which is accompanied by a rearrangement of all quantum states. The rearrangement occurs always when the flux trapped in the $\mathrm{N}$ layer changes by $\Phi_{0}=h c / 2 e$.

To conclude, we note that the mean free path of the quasiparticles was expected to be the largest parameter of the problem. Allowance for the scattering by impurities will decrease the amplitude of the resonance spikes.

\section{Conclusions}

A theory of coherent phenomena in simply connected cylindrical normal conductors with a mesoscopic-scale cross section has been developed in this study. It is assumed that the normal metal is pure and coated with a thin superconducting layer with a thickness of the order of the coherence length $\xi_{0}$, and that their electric contact is good. Under these conditions the quasiparticles with energy $E<\Delta$ experience Andreev scattering at the NS boundaries. They move ballistically through the normal metal in the field of the vector potential excited by a narrow solenoid at the center of the conductor. On a single circuit of a quasiparticle along the perimeter of the cylinder cross section, the wave function phase of a «particle» («hole») changes by $\pm \frac{e}{h c} \oint_{\mathcal{L}} \mathbf{A} d \mathbf{s}$. This integral is equal to the flux $\Phi$ through the surface bounded by the trajectory $\mathcal{L}$. In the field $\mathbf{A}$ excited by the solenoid, the flux $\Phi$ is a constant independent of the trajectory shape. The sensitivity to the vector potential field is identical for all the quantum states of the quasiparticles.

If a simply connected cylinder is placed in a weak magnetic field, the situation becomes more complicated. For different quantum states of the quasiparticle the increment in the wave function phase is determined by the flux $\Phi$, which depends on the shape of the trajectory. As follows from studies of quantum coherent phenomena in normal conductors with specular reflection of electrons at the cylinder boundary [8], the universal period $h c / e$ of the flux oscillations of the thermodynamic quantities is only connected with the states localized near the cylinder boundary.

This study is confined to the effect of the vector potential field on the phase of quasiparticle wave functions, which permits us to investigate the shapes of the quasiclassical trajectories of «particles» and «holes». The quasiparticles experience Andreev scattering at the NS boundaries. As a result, a set of Andreev levels appear, which determine the behavior of the thermodynamic quantities of the structure. Within the multidimensional quasiclassical method, a dispersion relation was obtained, which can be used to find the spectrum of Andreev levels. Expanding it in a series in the parameter $1 / k_{F} R<<1$ (the quasiclassical parameter is $k_{F} R>>1$ ) and keeping only the zero-order terms in the equation, we obtain Eq. (34) for the energy near the Fermi level, which bears resemblance to Kulik's spectrum for an SNS contact [48]. The expression describes the states of the quasiparticles inside the cylinder cross section when they move along the chord connecting two points at the NS boundary of the sample. The chord length depends on the radial angle $\alpha$ at which both the points are visible. The product of this angle and the trapped flux has the meaning of the phase difference of the contact. Using the spectrum of Eq. (34), the density of states $v(E)$ is calculated as a function of the flux $\Phi$, which displays a stepwise behavior. Whenever the flux increases by the value $\Phi_{0}$, the number of steps increases by one. If the density of states is differentiated with respect to the flux $\Phi$, a set of resonance spikes with a period equal to a superconducting flux quantum appears.

\section{Acknowledgment}

The authors are indebted to A. Kadigrobov for helpful discussions. One of the authors (G. A. G. ) thanks the Department of Applied Physics, Chal- 
mers University of Technology and Göteborg University for the invitation to participate in joint research at Göteborg and for warm hospitality. Financial support from the Swedish KVA is gratefully acknowledged. G. A. G. would like to thank A. N. Omelyanchouk for discussions and reading of this manuscript.

1. J. Bardeen, L. N. Cooper, and J. R. Schrieffer, Phys. Rev. 108, 1175 (1957).

2. B. S. Deaver, Jr. and W. M. Fairbank, Phys. Rev. Lett. 7, 43 (1961)

3. R. Doll and M. Nabauer, Phys. Rev. Lett. 7, 51 (1961).

4. F. London, Superfluids, I, Dover Publications, Ins. (1950).

5. Y. Aharonov and D. Bohm, Phys. Rev. 115, 485 (1959).

6. C. N. Yang, Rev. Mod. Phys. 34, 694 (1962).

7. I. O. Kulik, Pis'ma Zh. Eksp. Teor. Fiz. 11, 407 (1970) [JETP Lett. 11, 275 (1970)].

8. E. N. Bogachek and G. A. Gogadze, Zh. Eksp. Teor. Fiz. 63, 1839 (1972) [Sov. Phys. JETP 36, 973 (1973)]

9. T. W. Nee and R. E. Prange, Phys. Lett. A25, 582 (1967).

10. E. N. Bogachek and G. A. Gogadze, Pis'ma Zh. Eksp. Teor. Fiz 17, 169 (1973) [JETP Lett. 17, 115 (1973)].

11. E. N. Bogachek and G. A. Gogadze, Zh. Eksp. Teor. Fiz. 67, 621 (1974) [Sov. Phys. JETP 40, 308 (1974)].

12. E. N. Bogachek, G. A. Gogadze, and I. O. Kulik, Fiz. Nizk. Temp. 2, 461 (1976) [Sov. J. Low Temp. Phys. 2, 228 (1976)].

13. G. A. Gogadze, Solid State Commun. 49, 277 (1984)

14. G. A. Gogadze, Fiz. Nizk. Temp. 91051 (1983) [Sov. J. Low Temp. Phys. 9, 543 (1983)].

15. E. N. Bogachek, G. A. Gogadze, and I. O. Kulik, Phys. Status Solidi B67, 287 (1975).

16. W. A. Little and R. D. Parks, Phys. Rev. Lett. 9, 9 (1962).

17. R. D. Parks and W. A. Little, Phys. Rev. 133, 97 (1964).

18. M. Tinkham, Phys. Rev. 129, 2413 (1963).

19. E. N. Bogachek, G. A. Gogadze, and I. O. Kulik, Fiz. Nizk. Temp. 4, 1156 (1978) [Sov. J. Low Temp. Phys. 4, 544 (1978)].

20. N. B. Brandt, D. V. Gitsu, A. A. Nikolaeva, and Ya. G. Ponomarev, Pis'ma Zh. Eksp. Teor. Fiz. 24, 304 (1976) [JETP Lett. 24, 272 (1976)].

21. N. B. Brandt, D. V. Gitsu, A. A. Nikolaeva, and Ya. G. Ponomarev, Zh. Eksp. Teor. Fiz. 72, 2332 (1977) [Sov. Phys. JETP 45, 1226 (1977)].

22. N. B. Brandt, E. N. Bogachek, D. V. Gitsu, G. A. Gogadze, I. O. Kulik, A. A. Nikolaeva, and Ya. G. Ponomarev, Fiz. Nizk. Temp. 8, 718 (1982) [Sov. J. Low Temp. Phys. 8, 358 (1982)].

23. I. O. Kulik, Fiz. Nizk. Temp. 13, 206 (1987) [Sov. J. Low Temp. Phys. 13, 115 (1987)]

24. Y. Imry, in: Directions in Condensed Matter Physics, G. Grinstein and G. Mazenko (eds.), World Scientific, Singapore (1986), p. 101

25. R. A. Webb, S. Washburn, C. P. Umbach, and R. B. Laibowitz, Phys. Rev. Lett. 54, 2696 (1985).
26. V. Chandrasekhar, N. Rooks, S. Wind, and D. Prober, Phys. Rev. Lett. 55, 1610 (1985).

27. B. L. Altshuler, A. G. Aronov, and B. Z. Spivak, Pis'ma Zh. Eksp. Teor. Fiz. 33, 101 (1981) [JETP Lett. 33, 97 (1981)].

28. D. Yu. Sharvin and Yu. V. Sharvin, Pis'ma Zh. Eksp. Teor. Fiz. 34, 285 (1981) [JETP Lett. 34, 272 (1981)].

29. B. L. Altshuler, A. G. Aronov, B. Z. Spivak, D. Yu. Sharvin, and Yu. V. Sharvin, Pis'ma Zh. Eksp. Teor. Fiz. 35, 476 (1982) [JETP Lett. 35, 475 (1982)].

30. B. L. Altshuler and A. G. Aronov, in: Electron-Electron Interaction in Disordered Systems, A. L. Efros and M. Pollak (eds.), North-Holland, Amsterdam (1985), p. 1.

31. S. Washburn and R. A. Webb, Adv. Phys. 35, 375 (1986).

32. A. G. Aronov and Yu. V. Sharvin, Rev. Mod. Phys. 59, 755 (1987).

33. A. A. Zvyagin and I. V. Krive, Fiz. Nizk. Temp. 21, 687 (1995) [Low Temp. Phys. 21, 533 (1995)].

34. E. N. Bogachek, Fiz. Nizk. Temp. 2, 473 (1976) [Sov. J. Low Temp. Phys. 2, 235 (1976)].

35. C. W. J. Beenakker and H. van Houton, Phys. Rev. B38, 3232 (1988).

36. P. H. M. van Loosdrecht, C. W. J. Beenakker, H. van Houton, J. G. Williamson, B. J. van Wees, J. E. Mooij, C. T. Foxon, and J. J. Harris, Phys. Rev. B38, 10162 (1988).

37. B. Müller-Allinger and A. C. Mota, Phys. Rev. Lett. 84, 3161 (2000).

38. P. Visani, A. C. Mota, and A. Pollini, Phys. Rev. Lett. 65, 1514 (1990).

39. A. Lodder and Yu. V. Nazarov, Phys. Rev. B58, 5783 (1998).

40. A. F. Andreev, Zh. Eksp. Teor. Fiz. 46, 1823 (1964) [Sov. Phys. JETP 19, 1228 (1964)].

41. A. Kadigrobov, A. Zagoskin, R. I. Shekhter, and M. Jonson, Phys. Rev. B52, R8662 (1995).

42. H. A. Blom, A. Kadigrobov, A. M. Zagoskin, R. I. Shekhter, and M. Jonson, Phys. Rev. B57, 9995 (1998).

43. P. G. de Gennes, Superconductivity of Metals and Alloys, Benjamin, New York (1966).

44. C. Caroli and J. Matricon, Phys. Kond. Materie 3, 380 (1965).

45. C. Caroli, P. G. de Gennes, and J. Matricon, Phys. Lett. 9, 307 (1964).

46. J. B. Keller and S. I. Rubinow, Ann. Physics 9, 24 (1960)

47. J. B. Keller, Ann. Physics 4, 180 (1958).

48. I. O. Kulik, Zh. Eksp. Teor. Fiz. 57, 1745 (1969) [Sov. Phys. JETP 30, 944 (1969)].

49. Yu. K. Dzhikaev, Zh. Eksp. Teor. Fiz. 68, 295 (1975) [Sov. Phys. JETP 41, 144 (1975)].

50. L. Yu. Gorelik and A. M. Kadigrobov, Fiz. Nizk. Temp. 7, 131 (1981) [Sov. J. Low Temp. Phys. 7, 65 (1981)].

51. A. Kadigrobov, R. Shekhter, and M. Jonson, Physica B218, 134 (1996).

52. J. Bardeen, R. Kümmel, A. E. Jacobs, and L. Tewordt, Phys. Rev. 187, 556 (1969) 